\title{
Fabrication and Characterization of Pulp/Chitosan Composite Membranes Crosslinked with 3-Methylglutaric Anhydride for Pervaporation of Ethanol/Water Mixture
}

\author{
Truong Thi Cam Trang, Nguyen Thi Nhung, Takaomi Kobayashi \\ Department of Materials Science and Technology, Nagaoka University of Technology, Kamitomioka, \\ Nagaoka, Japan \\ E-mail:takaomi@nagaokaut.ac.jp \\ Received September 29, 2010; revised October 22, 2010; accepted November 8, 2010
}

\begin{abstract}
Chitosan/Cellulose (CTS/CL) composite membranes were prepared by cross-linking reaction with 3-methylglutaric anhydride (3MGA). The cross-linked membranes with CTS/CL were obtained at different CTS contents in variations from 50 to $100 \mathrm{wt} \%$, and these membranes were applied in the dehydration of ethanol/water mixtures. Especially, it was observed that in the case of a composite membrane containing chitosan $80 \%$ (CTS/CL-80/20) showed a performance with a separation factor of $\alpha=17.1$ and a total permeation flux of J $=326 \mathrm{~g} /\left(\mathrm{m}^{2} \mathrm{~h}\right)$. It was observed that the total permeation flux decreased when the cross-linking increased and the increase in the ethanol content in the feed solution showed an increase in the separation factor. The CTS/ CL-80/20 showed excellent performance with good mechanical strength and dehydration performance in the ethanol/water mixture separation.
\end{abstract}

Keywords: Pervaporation, Chitosan, Cellulose, Contact Angle, Ethanol, Composite Membrane

\section{Introduction}

It is known that pervaporation (PV) is a potential method for separation of liquid mixtures in membrane processes. It is used for dehydration process of mixtures with organic components which have close boiling points. PV has been proven as a highly efficient separation process [1-4]. In recent years, a major advantage of this process is the ability to separate azeotropic mixtures as an economical and simple alternative to conventional energy-intensive technologies, especially water-ethanol systems [5]. As an important application in industrial dehydration processes, several PV studies have been reported for the separation of aqueous/organic mixtures [6-11] using hydrophilic dense membranes. Among them, blended polymer membranes have been extensively studied resulting in increased flux and selectivity values. For example, the blended membranes of two oppositely charged polymers formed high density membrane structure and could result in high selectivity in the membranes [12]. In blended polyelectrolyte membranes for PV dehydration of alcohols, blended chitosan and polyacrylic acid membranes have been used [12,13], as well as blended chitosan and sodium al- ginate membranes [14]. It was furthermore studied that synthetic polymer membranes were also widely applied in PV separation [15-19]. We noted that, by the application of such blending techniques, the properties of the resultant polymer membranes could demonstrated superior changes in the intrinsic chemical, physical, mechanical, and morphological properties, as compared to non blended membranes. In addition, as biopolymers are being used, CTS is a material that can be found in crustacean shells, which is usually wasted from seafood industries. Therefore, as a suitable biopolymer, their applications for blended and composite membranes have been reported [12-14]. It is known that CTS is a cyclo-aliphatic polymer that contains both active amino groups and hydroxyl groups (Figure 1). By using CTS cross-linked membranes by several chemicals such glutaraldehyde [17,20-22], formaldehyde [16,19,23,24], sulfuric acid $[19,23,25]$. Therefore, the CTS membranes PV performance in blended chitosan and polyvinyl alcohol membranes for dehydration of isopropanol was investigated [26].

On the other hand, cellulose (CL) which usually has high molecular weights due to long chains of d-glucose 


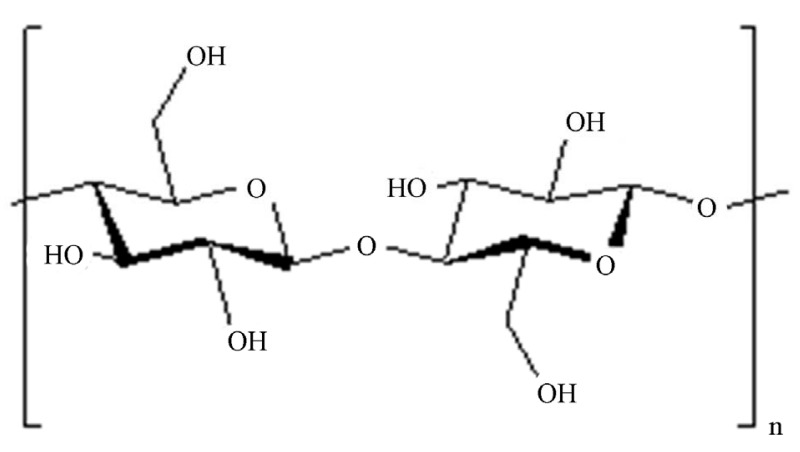

(a)

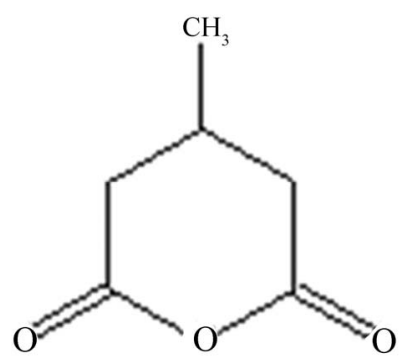

(b)

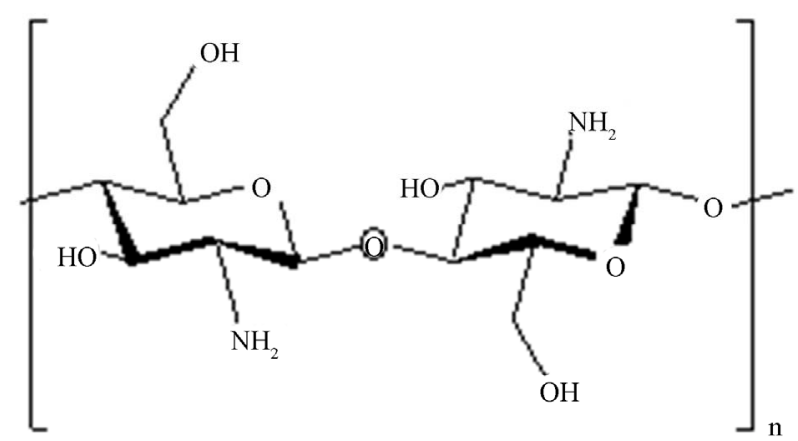

(c)

Figure 1. Schematic procedure of preparation of CTS/CL membrane.

units joined together by $\beta$-1,4-glucosidic bonds (Figure 1) is the most widely available organic biopolymer. It is nontoxic, renewable, biodegradable and modifiable to have great potential as an excellent industrial material $[27,28]$. More recently in PV processes, we have reported that vulcanized cellulose membranes showed very good dehydration performance in alcohol/water mixtures [Truong T. Cam Trang, Kobayashi Takaomi: Journal of Applied Polymer Science, DOI \#33258]. Due to such excellent properties of CL membrane, the present work addersses the continuing efforts to develop new biopolymer membranes for PV separation of aqueous-organic mixtures by combining both CTS and CL in order to improve the membrane performance. In the present investigation, attempts to examine the applicability of CTS/CL composite by using the cross-linker 3-metylglutaric anhydride were made for the purpose of dehydrating ethanol/water mixtures. The present work also explores the effect of polymer swelling and sorption selectivity in addition to the PV performance by varying ethanol/water contents from 10 to $90 \mathrm{wt} \%$.

\section{Experimental}

\subsection{Materials}

Chitosan, having a degree of deacetylation of $80 \%$, was purchased from Wako (Osaka, Japan) and 3-methylglutaric anhydride was purchased from TCI, Tokyo, Japan. Cellulose was received from Hokuetsu Paper Mills (Tokyo, Japan). Ethanol was purchased from Nacalai Tesque. Inc. Water was deionized and distilled before use.

\subsection{Membrane Preparation}

Figure 2 shows the schematic procedure for the preparation of the CTS/CL membranes. The membranes were obtained as follows: CTS was dissolved in de-ionized water with $2 \mathrm{wt} \%$ acetic acid to be $1 \mathrm{wt} \%$ in concentration. The CTS solution was filtered in order to remove un-dissolved parts and impurities before preparation of the CTS/CL membranes. In the raw CL $(15 \mathrm{~g}), 120 \mathrm{ml}$ of water were mixed and the mixture was crushed using a mixer stirring at $250 \mathrm{rpm}$. After the process was finished, the CL water mixture were mixed with the CTS solution under stirring at $250 \mathrm{rpm}$ and kept for $36 \mathrm{~h}$ in order to be sure it was completely homogeneous. For the cross-linking process, $5 \mathrm{ml}$ of $5 \mathrm{wt} \% 3 \mathrm{MGA}$ in de-ionized water was added and mixed. The CTS/CL solution was poured into a flat glass plate and the CTS/CL membranes were formed after dried at $40^{\circ} \mathrm{C}$ for $36 \mathrm{~h}$ to then be removed from the glass plate. The membranes were washed and rinsed repeatedly with de-ionized water and then dried at room temperature for $24 \mathrm{~h}$. Six different membranes for the CTS/CL mixtures were prepared as weight ratio of $100 / 0,85 / 15,80 / 20,75 / 25,60 / 40$ and $50 / 50$ and were denominated as CTS/CL-100/0, CTS/CL-85/15, CTS/CL80/20, CTS/CL-75/25, CTS/CL-60/40 and CTS/CL-50/50, respectively. The thickness of each membrane was measured using a micrometer screw gauge and found to be of about $100-150 \mu \mathrm{m}$.

\subsection{CTS/CL Membranes Characterization}

In order to characterize the CTS/CL membranes, scanning electron microscopy (SEM) was used to observe the morphology of the surface and the cross-section of the resultant membranes. Samples of CTS/CL were fractured in liquid nitrogen before the SEM measurements. The 


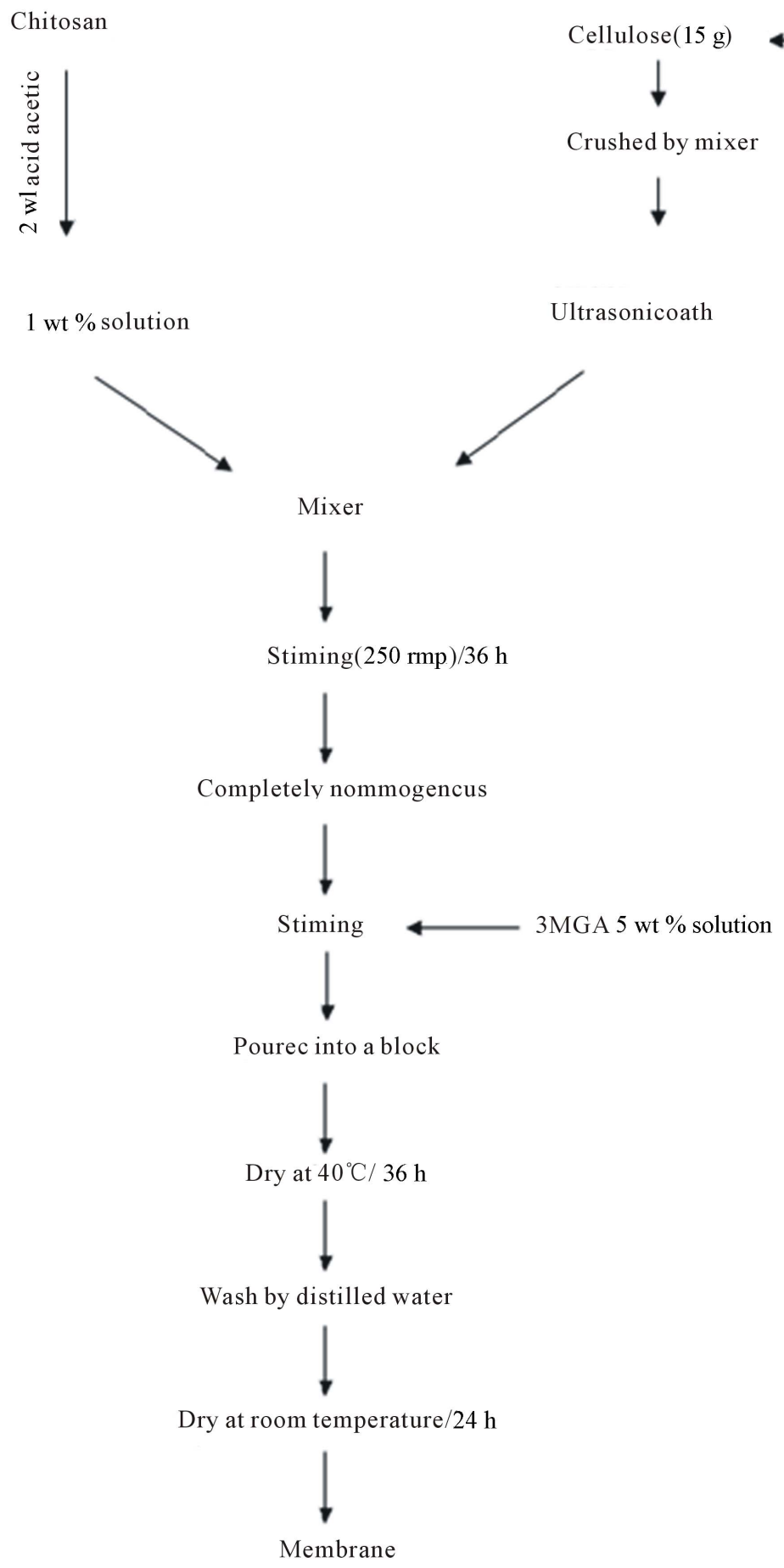

Figure 2. Structure of (a) Cellulose; (b) 3-methylglutaric anhydride and (c) Chitosan.

fractured section of the CTS/CL membrane was coated with a gold conductive sputtered layer. The surface and the cross-section of CTS/CL membranes were observed by a JSM-5300LV (JEOL, Japan).

The tensile strength of the CTS/CL membranes was measured using a LTS-500N-S20 (Minebea, Japan). Cross-sectional area of samples of known width $(10 \mathrm{~mm})$ and thickness $(0.1 \mathrm{~mm})$ were used in the calculations. The value of the tensile strength was calculated by using the following equation:

$$
\text { tensile strength }=\frac{\text { max imum load }}{\text { cross-sectional area }}\left(\mathrm{N} / \mathrm{mm}^{2}\right)
$$

Chemical properties of the CTS/CL membranes were 
analyzed by FT-IR method. All of FT-IR spectra were obtained by using a FT-IR spectrophotometer (Model8201; Shimadzu Corp) in transmission mode.

Surface areas of the CTS/CL membranes were measured using a Flowsorb 2300 (Shimadzu, Japan) and contact angle of the aqueous ethanol solution on the membrane surface was determined using a DropMaster100 contact angle meter (Kiowa Interface Science Co. LTD) at room temperature.

For observing the swelling of the CTS/CL membranes, dried membranes were immersed in $10 \mathrm{wt} \%$ to $90 \mathrm{wt} \%$ of ethanol aqueous solution in a sealed vessel at room temperature for $96 \mathrm{~h}$. The membranes were taken out of the solution rapidly, wiped with filter paper and then measured in the length and thickness quickly in addition with its weight. Solvent content was determined from the difference in the weight of the membranes before and after immersion in aqueous solutions. The solvent content (SC) was expressed as:

$$
\mathrm{SC}=\left[\left(\mathrm{W}_{s}-\mathrm{W}_{d}\right) / \mathrm{W}_{\mathrm{d}}\right] \times 100 \%
$$

where $\mathrm{W}_{\mathrm{d}}$ and $\mathrm{W}_{\mathrm{s}}$ denote the weight of the films before and after immersion in the solution, respectively.

The degree of swelling (DS) of the CTS/CL membranes was calculated by comparing the volume of the membranes before $\left(\mathrm{V}_{\mathrm{b}}\right)$ and after $\left(\mathrm{V}_{\mathrm{a}}\right)$ immersion in the solution:

$$
\mathrm{DS}=\mathrm{V}_{a} / \mathrm{V}_{\mathrm{b}}
$$

\subsection{Pervaporation Experiments}

A schematic diagram of the PV apparatus is shown in Figure 3. The permeation experiment was performed by using a common film cell, which was connected using a magnetic holder to fit the films $(\Phi=46 \mathrm{~mm})$ having an effective film area of $17 \times 10^{-4} \mathrm{~m}^{2}$. The membrane cell

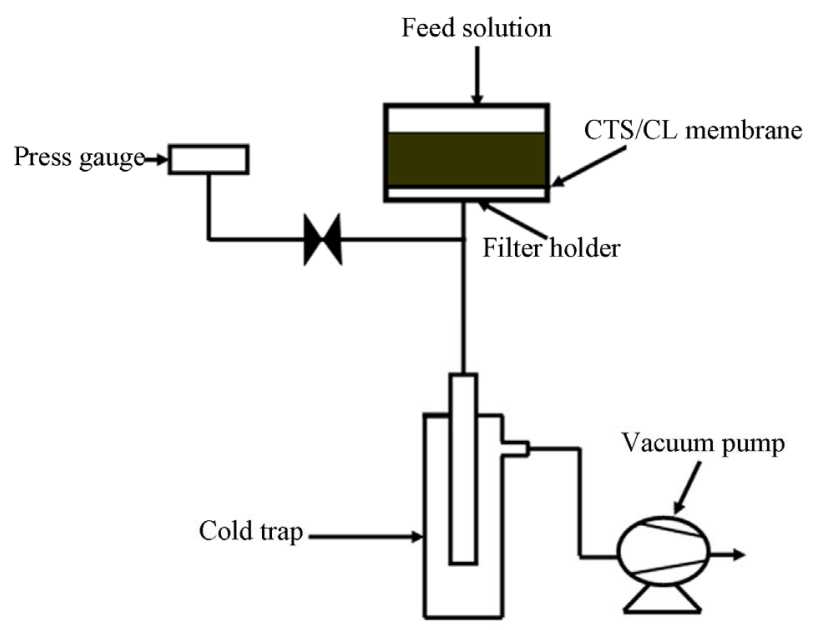

Figure 3. Schematic diagrams of the PV apparatus. inside was filled with alcohol/water solution up to 150 ml. Here, the solution was exposed to atmospheric pressure on the upper side and the solution amount was fed by addition of alcohol-water solution from the upper side by means of a feed pump. In the bottom of the membrane cell, a stainless tube having an inner diameter of $58 \mathrm{~mm}$ was connected to a cold trap and a vacuum pump (ULVAC, DA-20D, Tokyo, Japan). Then, the inside pressure was kept at $124( \pm 0.5) \mathrm{Pa}$ by using the vacuum pump through the cold trap. The inside pressure was measured with a pressure gauge (MKS Baratron, 122A). For the PV process, all the experiments were performed at $25^{\circ} \mathrm{C}$ and variable feed of alcohol in the range of 10 to $90 \mathrm{wt} \%$ in the feed solution. The permeated stream was condensed downstream and collected in a cold trap by freezing with liquid nitrogen after the pumping was started. The condensed sample was collected at a given period of time and then weighed. In order to evaluate alcohol and water concentrations in the permeated sample solution, a gas chromatography equipment (Shimadzu TC-14B) was used with Helium as the carrier gas in a pack column (Poly A-135) at $140^{\circ} \mathrm{C}$.

The permeability of the membranes for alcohol/water was calculated by the separation factor $(\alpha)$ defined as

$$
\alpha_{w / a}=\left(\mathrm{y}_{\mathrm{w}} / \mathrm{y}_{\mathrm{a}}\right) /\left(\mathrm{x}_{\mathrm{w}} / \mathrm{x}_{\mathrm{a}}\right)
$$

where $\mathrm{x}$ and $\mathrm{y}$ were the weight fractions of the species in the feed and the permeate, respectively. For the description of alcohol and water, the subscripts a and were used, respectively.

Permeate flux, J was calculated by using the following Equation (4)

$$
\mathrm{J}=\mathrm{Q} / \mathrm{At}
$$

where $Q(g)$ was the total mass of permeate for $t$ hours and $A\left(\mathrm{~m}^{2}\right)$ denoted the effective area $\left(17 \times 10^{-4} \mathrm{~m}^{2}\right)$ of the membrane.

\section{Results and Discussion}

\subsection{Characteristics of CTS/CL Membranes}

As mentioned in the experimental section, membranes consisting of CTS and CL were prepared with different amounts of CTS and CL for CTS/CL $=100 / 0,85 / 15$, $80 / 20,75 / 25,60 / 40$ and 50/50. Each membrane was cross-linked using 3MGA. Figure 4 shows the resultant SEM images of the CTS/CL membranes with and without the cross-linking treatment. In the pictures (Figures 4(g) and (h)), the cross section of the obtained membranes had about $120 \mu \mathrm{m}$ thickness in the untreated membrane consisting of $80 / 20$ of the CTS/CL. It was clear that the membrane presented many cellulose fibers having about $10 \mu \mathrm{m}$ in diameter. It could be observed that the morphological structure of the surfaces of the 

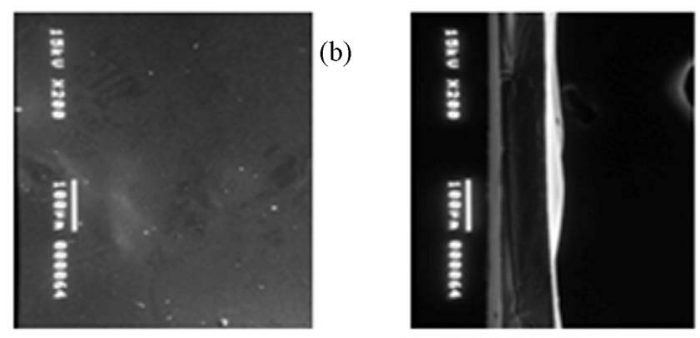

(a)

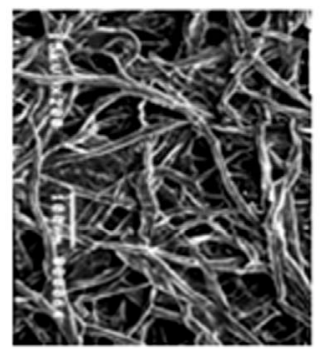

(d)

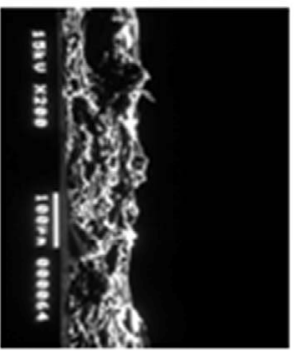

(c)
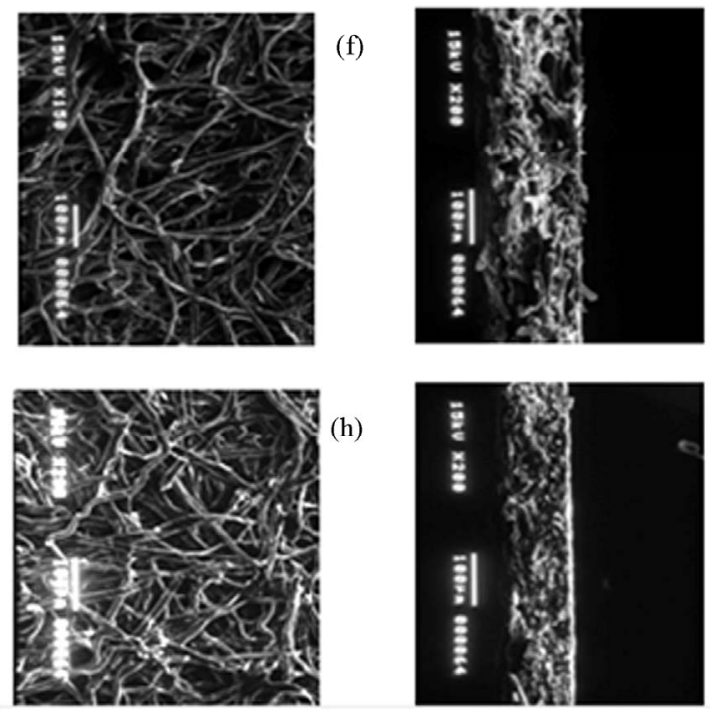

(g)

(e)

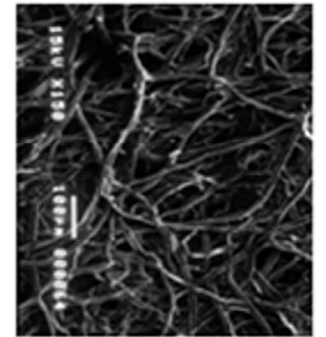

(h)

Figure 4. SEM images of CTS/CL blended membranes: (a) surface of membrane CTS/CL-100/0; (b) Cross-section of membrane CTS/CL-100/0; (c) Surface of membrane CTS/CL-80/20; (d) Cross-section of membrane CTS/CL-80/20; (e) Surface of membrane CTS/CL-50/50; (f) Cross-section of membrane CTS/CL-50/50; (g) Surface of CTS/CL-80/20 mem-brane without cross-linking; (h) Cross section of CTS/ CL-80/20 membrane without cross-linking.

four membranes in the pictures $(\mathrm{a} \sim \mathrm{h})$ was obtained with the cross-linking treatment and had a difference in the membrane thickness and number of CL fibers. With the increasing in the cellulose contents in the CTS/CL membranes, the cross-section which was of lower cellulose content became denser.

Figure 5 shows the FT-IR spectra of CL sample (a), CL/3MGA sample (b), CTS sample (c), CTS/3MGA sample (d), CTS/CL-80/20 without cross-linking treatment, (f) CTS/ CL-80/20 with cross-linking treatment. In the FT-IR spectrum (a) of CL had the IR bands of OH

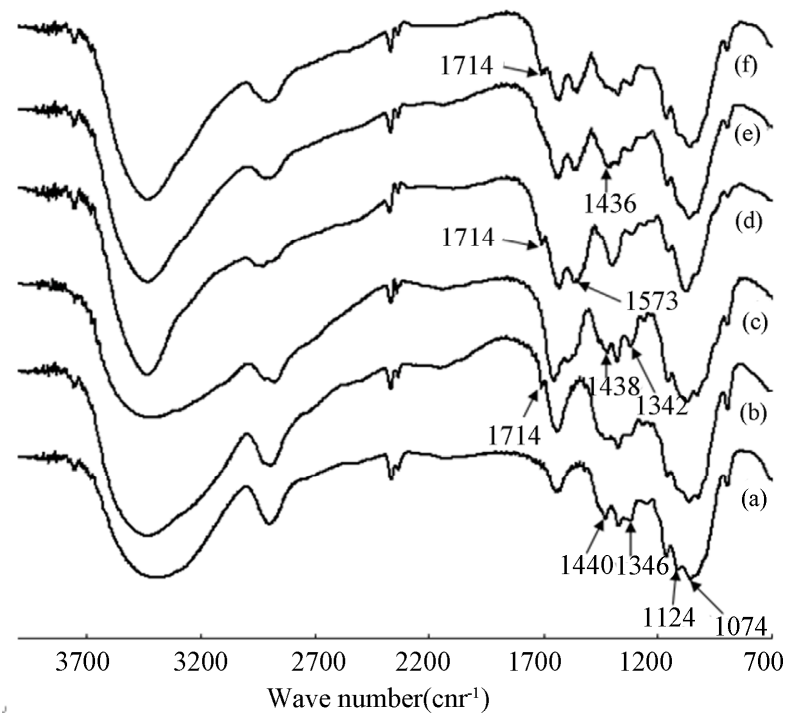

Figure 5. IR-FR spectra of the CTS/CL blended membranes.

stretching at 3425, 1664, 1346 and $1124 \mathrm{~cm}^{-1}, 2924$, 1382 and $906 \mathrm{~cm}^{-1}$ of $\mathrm{CH}$ stretching, $1440 \mathrm{~cm}^{-1}$ of $\mathrm{CH}_{2}$ blending, 1172 and $1074 \mathrm{~cm}^{-1}$ of C-O-C stretching were confirmed $[26,29]$. In the (b), the FT-IR spectrum of the CL/3MGA membrane treated by cross-linked showed that, the appearance of IR bands at $1714 \mathrm{~cm}^{-1}(\mathrm{C}=\mathrm{O})$ and the absence of the bands at $1440 \mathrm{~cm}^{-1}\left(\mathrm{CH}_{2}\right), 1346$ and $1124 \mathrm{~cm}^{-1}(\mathrm{OH})$ could be confirmed. In the spectrum of CTS (c), the characteristic absorption bands were observed at 3448 and $1332 \mathrm{~cm}^{-1}(\mathrm{OH}), 1670 \mathrm{~cm}^{-1}$ (amide I), $1587 \mathrm{~cm}^{-1}$ (amide II), $1438 \mathrm{~cm}^{-1}\left(-\mathrm{CH}_{2}\right.$ bending), $1386 \mathrm{~cm}^{-1}(\mathrm{CH}), 1160 \mathrm{~cm}^{-1}(\mathrm{C}-\mathrm{O}-\mathrm{C}), 1090$ and $1040 \mathrm{~cm}^{-1}$ (C-O stretching). In a similar manner to the $\mathrm{CL} / 3 \mathrm{MGA}$ sample, the cross-linked treatment of 3MGA was carried out in the CTS/3MGA sample for (d). The IR bands at $1714 \mathrm{~cm}^{-1}(\mathrm{C}=\mathrm{O}), 1573 \mathrm{~cm}^{-1}$ (Amide II) and the absence of the bands at $1438 \mathrm{~cm}^{-1}$ $\left(-\mathrm{CH}_{2}\right)$ and $1332 \mathrm{~cm}^{-1}(\mathrm{OH})$ could be confirmed in the spectrum. For the 3MGA treatment, the membranes had IR bands at $1717 \mathrm{~cm}^{-1}$ for the $\mathrm{C}=\mathrm{O}$ group of 3MGA. This seemed to be explained due to the cross-linking reaction forming ester groups of 3MGA and $\mathrm{OH}$ groups of CL occurred in the CTS/ 3MGA membranes. Furthermore, (e) shows the FT-IR spectrum of the CTS/CL membrane. When a comparison was made between CL (a) and CTS (c), the IR bands presented a shift in the amide I and amide II of the CTS from $1670 \mathrm{~cm}^{-1}$ and $1587 \mathrm{~cm}^{-1}$ to 1651 and $1576 \mathrm{~cm}^{-1}$, respectively. Also, the disappearance of band at $1124 \mathrm{~cm}^{-1}(\mathrm{OH})$ was observed. Moreover, in the CTS/CL/ 3MGA membrane (f), both $1651 \mathrm{~cm}^{-1}$ (amide I) and $1576 \mathrm{~cm}^{-1}$ (amide II) bands were observed in addition with the appearance of $1714 \mathrm{~cm}^{-1}$ for the $\mathrm{C}=\mathrm{O}$ of $3 \mathrm{MGA}$ and the disappearance of $1124 \mathrm{~cm}^{-1}$ for OH group of CTS and CL. 
As characteristic of these CTS/CL membranes, the value of tensile strength and elongation of the membrane are given in Table 1. From these results, it could be observed that the CTS/CL-80/20 membrane exhibited high tensile strength of $89.2 \mathrm{~N} / \mathrm{mm}^{2}$. With the increase of the $\mathrm{CL}$ contents in the CTS/CL membranes, the CTS/CL membranes became brittle. Table 1 also shows the surface area and the contact angle of water for the CTS/CL membranes. There was tendency for the CTS/CL membranes to show a high surface area at CTS/CL $=80 / 20$. The data of the contact angle decreased with the increase in the weight of ratio CTS/CL. This was due to a high affinity of the water molecules with the CTS segments in the CTS/CL membrane.

\subsection{Separation of Ethanol and Water by PV Process}

The separation performance of ethanol/water mixtures by the CTS/CL membranes was investigated using PV apparatus (Figure 3). In the dehydration process of the mixture, the feed composition of ethanol and water was changed in the range of $10 \mathrm{wt} \%$ to $90 \mathrm{wt} \%$ of ethanol. Then the total permeation flux and separation factor was obtained. In order to understand clearly the PV characteristics, the effect of ethanol composition on the total permeation flux was determined in Figure 6(a). As the ethanol concentration in the feed solution was increased, the value of the total permeation flux considerably decreased. Especially, the tendency of the decline was more significant between CTS/CL $=80 / 20$ and $85 / 15$. When the CL content was increased in the films, there was a tendency for the values of total permeation flux to increase.

Figure 6(b) shows separation factor $(\alpha)$ for each CTS/ $\mathrm{CL}$ membrane. When the ethanol concentration increased from 10 to $90 \mathrm{wt} \%$, there was a tendency for the value of $\alpha$ to increase from 2.1 to 5.9 for the CTS/CL-75/25, from 5.6 to 17.1 for CTS/CL-80/20 and from 5.8 to 17.3 for the CTS/CL-85/15. In the CTS/CL-100/0 membrane the values were 5.9 and 17.9 at $10 \mathrm{wt} \%$ and $90 \mathrm{wt} \%$ of ethanol solution. It was noted that a reverse tendency could be seen in the data of the permeation flux and $\alpha$. With the increase in ethanol concentration in the feed so- lution, the separation factor increased, but also lower permeation flux is obtained. In the case of the CTS/CL without cross-linking treatment, the mechanical properties were not good for the PV experiments, but the value of the faster total permeation flux was observed in the

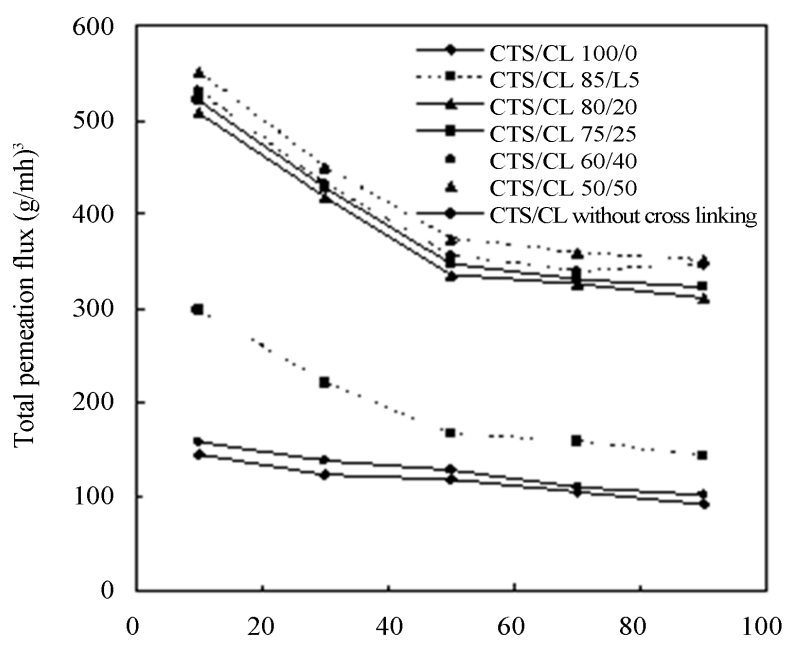

(a)

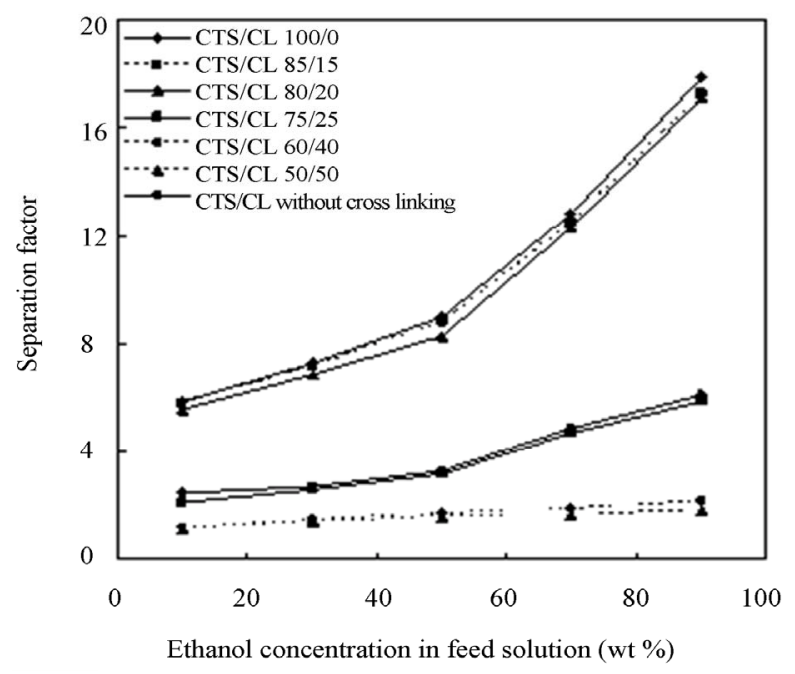

(b)

Figure 6. Effect of ethanol concentration on (a) Total permeation flux; (b) Separation factor.

Table 1. Physical properties of CTS/CL membranes.

\begin{tabular}{|c|c|c|c|c|c|}
\hline & Thickness $(\mu \mathrm{m})$ & Tensile strength $(\mathrm{N} / \mathrm{mm} 2)$ & Elongation(\%) & Surface area $(\mathrm{m} 2 / \mathrm{g})$ & Contact angle (degree) \\
\hline CTS/CL-100/0 & 100 & 55.8 & 7.6 & 78.0 & 80.2 \\
\hline CTS/CL-80/20 & 120 & 89.8 & 4.5 & 98.0 & 60.4 \\
\hline CTS/CL-75/25 & 120 & 55.6 & 4.7 & 72.0 & 52.7 \\
\hline CTS/CL-60/40 & 120 & 25.6 & 25.5 & 45.0 & 43.5 \\
\hline CTS/CL-50/50 & 120 & 24.3 & 27.1 & 43.2 & 47.6 \\
\hline CTS/CL without cross linking & 120 & 21.5 & 23.1 & 42.5 & 57.5 \\
\hline
\end{tabular}


range $90 \sim 165 \mathrm{~g} / \mathrm{m}^{2} . h$ and the separation factor was shown in the range of $2 \sim 6.2$.

As plotted in Figure 7, both the relationship of the total permeation flux and separation factor is clearly indicated. That is, the increase of the total permeation flux led to a decline in the value of separation factor. Among them, it was noted that the CTS/CL-80/20 membrane showed a high PV performance compared to other CTS/ CL membranes, although the value of total permeation flux was $500 \mathrm{~g} / \mathrm{m}^{2} \mathrm{~h}$ and separation factor was about 18 . It was also indicated that, the PV performance of untreated cross-linked film and CTS/CL-100/0 film resuted in low total permeation flux and high separation factor.

In order to have further information of the resultant membranes, as shown in Figure 8, the effect of different ethanol concentrations on (a) the solvent content (SC) and (b) the degree of swelling (DS) were examined. The CTS/CL membranes showed a decrease in SC in all membranes when the ethanol concentration increased. This meant that, due to the affinity of the CTS/CL membrane for water, the increase of water adsorbed in the membrane was obtained. It also could be seen that, a high swelling of all the CTS/CL membranes was observed at lower ethanol concentrations. The tendency changed to become lower when the content of CTS was decreased. This resulted in a decrease of the CL content in the membrane, since the value of DS decreased. This fact might be due to the presence of hydroxyl groups in the CL and to be easily adsorbed with water molecules. As listed in in Table 1, the value of water contact angles also supported that the CTS/CL membranes containing lower CL contents showed water resistance. That is, the cellulose segments in the film behaved so that they increase the penetration of water molecules into the membranes.

In order to know the PV performance of the present

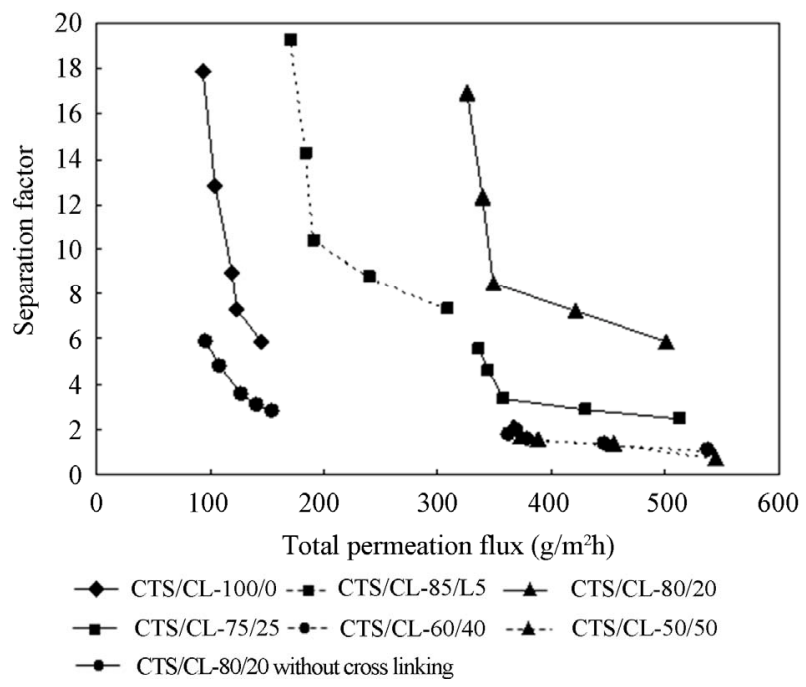

Figure 7. Plots of separation factor and total permeation flux.

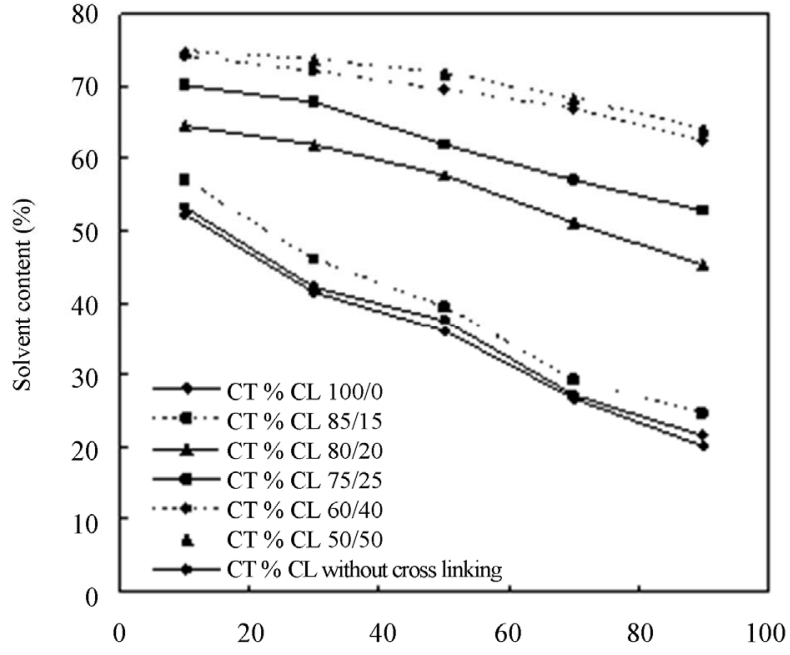

(a)

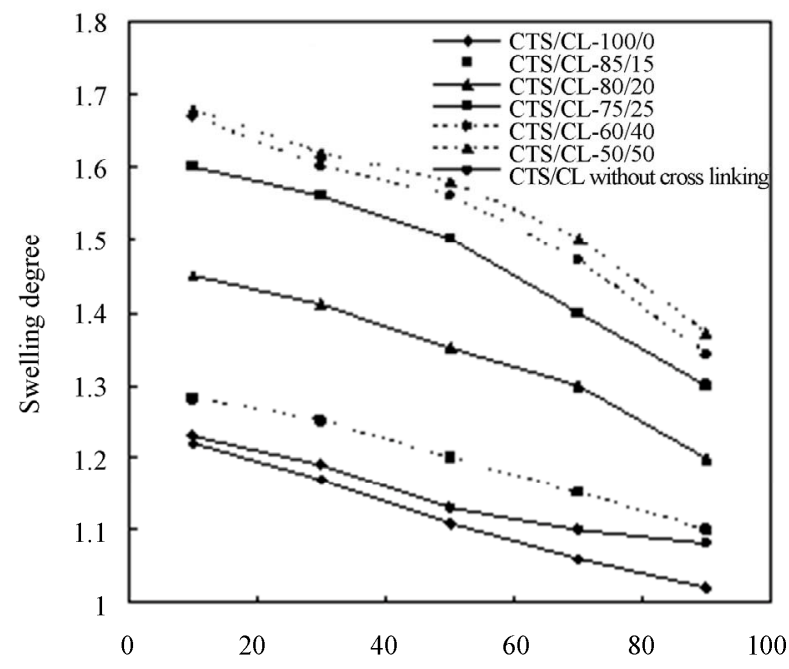

Ethanol concentration in feed solution (wt \%)

(b)

Figure 8. Solvent content (a) and degree of swelling (b) of the blended membranes observed at different ethanol concentration.

membranes, a comparison was made to the references for several polymeric membranes of (1) chitosan-silica complex [30] and (2) chitosan-HEC [31] and (3) chitosanpolyacrylic acid [10] in dehydration of ethanol. These processes of (1), (2) and (3) showed an excellent separation performance of $\alpha=234,10.5$ and 2216 with 234, 112 and $33 \mathrm{~g} /\left(\mathrm{m}^{2} \mathrm{~h}\right)$, for ethanol/water mixture, when the ethanol concentration was $90 \mathrm{wt} \%$. In the present work, the composite CTS/CL membrane showed permeable and separation performance with $326 \mathrm{~g} /\left(\mathrm{m}^{2} \mathrm{~h}\right)$ and $\alpha=$ 17.1 for the ethanol/water mixture. The CTS/CL membranes system in the present work therefore could dem- 
onstrate a higher permeation flux, even though the separation factor was somewhat lower than that of chitosanHEC blended film [31]. We also expected that the CTS/ CL membranes were totally made of CTS and CL which were typical biopolymers. This was of advantage in the case of CTS/CL membranes for sustainable materials and firstly reported in the present work. It could be concluded that the film of CTS and CL with 3-MGA treatment would become promising because of the proper alcohol concentration by PV process. Therefore, the CTS/CL biodegradable composite membranes were applicable with high permeation flux and stable operation for separation of ethanol/water mixtures in food and medical industrial uses.

\section{Conclusions}

In the present study, membranes of cellulose and chitosan were prepared with 3-methylglutaric anhydride as a cross-linking agent and applied for the PV experiments for the separation of ethanol/water mixtures. The separation process of ethanol/water mixtures was studied in different ethanol concentrations. The CTS/CL-80/20 membrane showed great performance in the PV process with a total permeation flux, $326 \mathrm{~g} / \mathrm{m}^{2} \mathrm{~h}$ by separation factor, 17.1. The CTS/CL membranes also showed a very good stability with benefits in the separation of azeotropic mixtures such as ethanol in aqueous solution by PV process.

\section{References}

[1] K. R. Lee, M. J. Liu and J. Y. Lai, "Pervaporation Separation of Aqueous Alcohol Solution through Asymmetric Polycarbonate Membrane," Separation Science and Technology, Vol. 29, No. 1, 1994, pp. 119-129. doi:10.1080/ 01496399408002472

[2] S. Takegami, H. Yamada and S. Tsujii, "Dehydration of Water Ethanol Mixtures by Pervaporation Using Modified Poly (Vinyl Alcohol) Membrane," Polymer Journal, Vol. 24, 1992, pp. 1239-1250. doi:10.1295/polymj.24. 1239

[3] K. M. Song and W. H. Hong, "Dehydration of Ethanol and Isopropanol Using Tubular Type Cellulose Acetate Membrane with Ceramic Support in Pervaporation Process," Journal of Membrane Science, Vol. 123, No. 1, 1997, pp. 27-35. doi:10.1016/S0376-7388(96)00198-6

[4] R. Y. M. Huang, "Membrane Separation Process," Elsevier Science, New York, 1991.

[5] R. Y. M. Huang, "Pervaporation Membrane Separation Process," Elsevier, Amsterdam, 1991.

[6] H. E. A. Bruschke, Japan Patent Kokai, Vol. 204, 1984, pp. 59-109.

[7] A. Pandey, C. R. Soccol, P. Nigam and V. T. Soccol, "Biotechnological Potential of Agro-Industrial Residues.
I: Sugarcane Bagasse," Bioresource Technology, Vol. 74, No. 1, 2000, pp. 69-80. doi:10.1016/S0960-8524(99)001 42-X

[8] B. Focher, M. T. Palma, M. Canetti, G. Torri, C. Cosentino, and G. Gastaldi, "Structural Differences between NonWood Plant Celluloses: Evidence from Solid State NMR, Vibrational Spectroscopy and X-Ray Diffractometry," Industrial Crops and Products, Vol. 13, No. 3, 2001, pp. 193-208. doi:10.1016/S0926-6690(00)00077-7

[9] S. Richardson and L. Gorton, "Characterisation of the Substituent Distribution in Starch and Cellulose Derivatives," Analytica Chimica Acta, Vol. 497, No. 1-2, 2003, pp. 27-65. doi:10.1016/j.aca.2003.08.005

[10] J. J. Shieh and R. Y. M. Huang, "Pervaporation with Chitosan Membranes II. On Polyelectrolyte Complexes of Chitosan and Polyacrylic Acid for the Separation of Ethanol-Water Mixtures," Journal of Membrane Science, Vol. 127, No. 2, 1997, pp. 185-202. doi:10.1016/S03767388(96)00279-7

[11] S. Y. Nam and Y. M. Lee, "Pervaporation and Properties of Chitosan/Poly (Acrylic Acid) Complex Membranes," Journal of Membrane Science, Vol. 135, No. 2, 1997, pp. 161-171. doi:10.1016/S0376-7388(97)00144-0

[12] X. Feng and R. Y. M. Huang, "Liquid Separation by Membrane Pervaporation: A Review," Industrial \& Engineering Chemistry Research, Vol. 36, 1997, pp. 1048. doi:10.1021/ie960189g

[13] Q. T. Nguyen, L. L. Blanc and J. Neel, "Preparation of Membranes from Poly (Acrylonitrile)-Poly (Vinyl Blends and the Study of Their Behavior in the Pervaporation of Water-Organic Liquid Mixtures," Journal of Membrane Science, Vol. 22, No. 2-3, 1985, pp. 245-255. doi:10. 1016/S0376- 7388(00)81284-3

[14] P. Kanti, K. Srigowri, J. Madhuri, B. Smitha and S. Sridhar, "Dehydration of Ethanol through Blend Membranes of Chitosan and Sodium Alginate by Pervaporation," Separation and Purification Technology, Vol. 40, No. 3, 2004, pp. 259 -266. doi:10.1016/j.seppur.2004.03.003

[15] L. G. Wu, C. L. Zhu and M. Liu, "Study of a New Pervaporation Membrane Part 1. Preparation and Characteristics of the New Membrane," Journal of Membrane Science, Vol. 90, No. 3, 1994, pp. 199-205. doi:10.1016/ 0376-7388(94)80071-5

[16] A. Chanachai, R. Jiraratananon, D. Uttapap, G. Y. Moon, W. A. Anderson and R. Y. M. Huang, "Pervaporation with Chitosan/Hydroxyethylcellulose (CS/HEC) Blended Membranes," Journal of Membrane Science, Vol. 166, No. 2, 2000, pp. 271-280. doi:10.1016/S0376-7388(99) 00269-0

[17] A. Chanachai, R. Jiraratananon, D. Uttapap, G. Y. Moon, W. A. Anderson and R. Y. M. Huang, "Pervaporation Dehydration of Ethanol-Water Mixtures with Chitosan/ Hydroxyethlcellulose (CS/HEC) Composite Membranes. I. Effect of Operating Conditions," Journal of Membrane Science, Vol. 195, No. 2, 2002, pp.143-151. doi:10.1016/ S0376-7388(01)00563-4

[18] C. Auclair, P. Schaetzel, R. Nobrega and C. Habert, "Dehydration of Light Oil by Pervaporation Using Poly 
(Vinyl Alcohol) Poly (Acrylic Acid-co-Maleic Acid) Membranes," Journal of Applied Polymer Science, Vol. 86, No. 7, 2002, pp. 1709-1716. doi:10.1002/app.11076

[19] J.-W. Rhim, S.-W. Lee and Y.-K. Kim, "Pervaporation Separation of Water-Ethanol Mixtures Using Metal-IonExchanged Poly (Vinyl Alcohol) (PVA)/Sulfosuccinic Acid (SSA) Membranes," Journal of Applied Polymer Science, Vol. 85, No. 9, 2002, pp.1867-1873. doi:10. 1002/app.10735

[20] H. A. Tsai, H. C. Chen, W. L. Chou, K. R. Lee and J. Y. Lai, "Pervaporation of Water/Alcohol Mixtures through Chitosan/Cellulose Acetate Composite Hollow-Fiber Membranes," Journal of Applied Polymer Science, Vol. 94, No. 4, 2004, pp. 1562-1568. doi:10.1002/app.20828

[21] M. Goto, A. Shiosaki and T. Hirose, "Separation of Water/Ethanol Vapor Mixtures through Chitosan and Crosslinked Chitosan Membranes," Separation Science and Technology, Vol. 29, No. 14, 1994, pp. 1915-1923. doi: $10.1080 / 01496399408002180$

[22] S. Suto and N. Ui, "Chemical Crosslinking of hydroxypropyl Cellulose and Chitosan Blends," Journal of Applied Polymer Science, Vol. 61, No. 13, 1996, pp. 22732278.

doi:10.1002/(SICI)1097-4628(19960926)61:13<2273::AI D-APP5>3.0.CO;2-9

[23] Y. J. Yin, K. D. Yao, G. X. Chang and J. B. Ma, "Properties of Polyelectrolyte Complex Films of Chitosan and Gelatin," Polymer International, Vol. 48, No. 6, 1999, pp. 429-432. doi:10.1002/(SICI)1097-0126(199906)48:6< 429::AID-PI160>3.0.CO;2-1

[24] J. X. Sun, F. Xu, Z. C. Geng, X. F. Sun and R. C. Sun, "Comparative Study of Cellulose Isolated by Totally Chlorine-Free Method from Wood and Cereal Straw," Journal of Applied Polymer Science, Vol. 97, No. 1, 2005, pp. 322-335. doi:10.1002/app. 21728
[25] C. L. Hsueh, J. F. Kuo, Y. H. Huang, C. C. Wang and C. Y. Chen, "Separation of Ethanol-Water Solution by Poly (Acrylonitrile-co-Acrylic Acid) Membranes," Separation and Purification Technology, Vol. 41, No. 1, 2005, 39-47. doi:10.1016/j.seppur.2004.04.002

[26] W. F. Guo, T. S. Chung and T. Matsuura, "Pervaporation Study on the Dehydration of Aqueous Butanol Solutions: A Comparison of Flux vs. Permeance, Separation Factor vs. Selectivity," Journal of Membrane Science, Vol. 245, No. 1-2, 2004, pp. 199-210. doi:10.1016/j.memsci.2004. 07.025

[27] N. D. Hilmioglu and S. Tulbentci, "Separation of IPA/ Water Mixtures by Pervaporation: Sorption and Pervaporation Results," Vacuum, Vol. 72, No. 1, 2003, pp. 35-40. doi:10.1016/S0042-207X(03)00096-4

[28] J. X. Sun, F. Xu, Z. C. Geng, X. F. Sun and R. C. Sun, "Comparative Study of Cellulose Isolated by Totally Chlorine-Free Method from Wood and Cereal Straw," Journal of Applied Polymer Science, Vol. 97, No. 1, 2005, pp. 322-335. doi:10.1002/app.21728

[29] R. C. Sun and J. Tomkinson, "Separation and Characterization of Cellulose from Wheat Straw," Separation Science and Technology, Vol. 39, No. 2, 2005, pp. 391411.

[30] Y. J. Yin, K. D. Yao, G. X. Chang and J. B. Ma, "Properties of Polyelectrolyte Complex Films of Chitosan and Gelatin," Polymer International, Vol. 48, No. 6, 1999, pp. 429-432. doi:10.1002/(SICI)1097-0126(199906)48:6< 429::AID-PI160>3.0.CO;2-1

[31] A. Chanachai, R. Jiraratananon, D. Uttapap, G. Y. Moon, W. A. Anderson and R. Y. M. Huang, "Pervaporation with Chitosan/Hydroxyethylcellulose (CS/HEC) Blended Membranes, Journal of Membrane Science, Vol. 166, No. 2, 2000, pp. 271-280. doi:10.1016/S0376-7388(99)0026 9-0 